Article

\title{
Broad Frequency Shift of Parametric Processes in Epsilon-Near-Zero Time-Varying Media
}

\author{
Vincenzo Bruno ${ }^{1}(1)$, Stefano Vezzoli ${ }^{2}$, Clayton DeVault ${ }^{3,4}$, Enrico Carnemolla ${ }^{5}$, \\ Marcello Ferrera $^{5}\left(\right.$, Alexandra Boltasseva ${ }^{3,4}\left(\right.$, Vladimir M. Shalaev ${ }^{3,4}(\mathbb{D}$, Daniele Faccio $1, *(\mathbb{C}$ \\ and Matteo Clerici ${ }^{6, *(D)}$ \\ 1 School of Physics and Astronomy, University of Glasgow, Glasgow G12 8QQ, UK; \\ v.bruno.1@research.gla.ac.uk \\ 2 The Blackett Laboratory, Department of Physics, Imperial College London, London SW7 2BW, UK; \\ s.vezzoli@imperial.ac.uk \\ 3 Purdue Quantum Science and Engineering Institute, Purdue University, 1205 West State Street, \\ West Lafayette, IN 47907, USA; cdevault@purdue.edu (C.D.); aeb@purdue.edu (A.B.); \\ shalaev@purdue.edu (V.M.S.) \\ 4 School of Electrical and Computer Engineering and Birck Nanotechnology Center, Purdue University, \\ 1205 West State Street, West Lafayette, IN 47907, USA \\ 5 Institute of Photonics and Quantum Sciences, Heriot-Watt University, Edinburgh EH14 4AS, UK; \\ enrico.carnemolla@st.com (E.C.); M.Ferrera@hw.ac.uk (M.F.) \\ 6 School of Engineering, University of Glasgow, Glasgow G12 8LT, UK \\ * Correspondence: daniele.faccio@glasgow.ac.uk (D.F.); matteo.clerici@glasgow.ac.uk (M.C.); \\ Tel.: +44-0141-330-5222 (M.C.)
}

Received: 29 November 2019; Accepted: 10 February 2020; Published: 15 February 2020

\begin{abstract}
The ultrafast changes of material properties induced by short laser pulses can lead to a frequency shift of reflected and transmitted radiation. Recent reports highlight how such a frequency shift is enhanced in spectral regions where the material features a near-zero real part of the permittivity. Here, we investigate the frequency shift for fields generated by four-wave mixing. In our experiment, we observed a frequency shift of more than $60 \mathrm{~nm}$ (compared to the pulse width of $\sim 40 \mathrm{~nm}$ ) in the phase conjugated radiation generated by a $500 \mathrm{~nm}$ aluminium-doped zinc oxide (AZO) film pumped close to the epsilon-near-zero wavelength. Our results indicate applications of time-varying media for nonlinear optics and frequency conversion.
\end{abstract}

Keywords: epsilon-near-zero media; four-wave mixing; frequency shift; transparent conductive oxides; optical Kerr nonlinearities; time-varying media

\section{Introduction}

Controlling the phase of an optical signal with a temporal resolution below one picosecond could prove essential for ultrafast signal processing. Such short time-scales can be achieved by exploiting nonlinear light-matter interaction in second-order nonlinear crystals (electro-optical effect) or Kerr effects in centrosymmetric media. In these processes, the instantaneous frequency is temporally modulated, and if the temporal phase change is uniform over the pulse duration, the optical spectrum can be rigidly shifted. This effect can be interpreted as time refraction [1,2], a phenomenon that has attracted the attention of the research community owing to its link to the dynamical Casimir effect and to Hawking radiation, as well as its implication in the formation of temporal band-gap structures and non-reciprocal devices [3-8]. Strong enhancement in light-matter interactions has been observed in the spectral region, where the real part of the dielectric permittivity $\left(\Re\left[\varepsilon_{r}\right]\right)$ of a medium approaches zero (ENZ) [9-11]. In the case of sub-wavelength thin films of transparent conductive 
oxide (TCO), such enhancement combined with an ultrafast response of the medium [12-23] results in the generation of phase conjugation (PC) and negative refraction (NR) with near-unity conversion efficiency [24]. One of the intriguing effects associated with the enhanced ultrafast material response is the observation of spectral shifts of the order of the pulse bandwidth $(10-15 \mathrm{~nm})$ for the transmitted and the reflected radiation in pump-and-probe experiments $[15,16]$. This phenomenon has been interpreted as originating from the adiabatic shift of the material refractive index enhanced, at the ENZ wavelength, by the associated slow-light condition [25].

In this work, we investigate the spectral shift of PC and NR in a time-varying TCO film (aluminium-doped zinc-oxide (AZO) [13]) at the ENZ wavelength. We report the efficient generation of a $>60 \mathrm{~nm}$ wavelength red-shifted PC and of both a red- and blue-shifted NR (covering nearly the same bandwidth, $\simeq 60 \mathrm{~nm}$ ) from a $500 \mathrm{~nm}$ thick AZO film pumped at the ENZ wavelength.

\section{Results}

Phase conjugation and negative refraction result from a four-wave mixing interaction mediated by a Kerr-type nonlinearity and satisfy a parametric amplification energy matching condition: $\omega_{\mathrm{PC}, \mathrm{NR}}=$ $2 \omega_{\mathrm{P}}-\omega_{\mathrm{S}}$, where $\omega_{\mathrm{p}}$ and $\omega_{\mathrm{S}}$ are the pump (P) and seed (S) frequencies, respectively [26-29]. The PC and NR are the processes satisfying the additional condition $\omega_{\mathrm{S}}=\omega_{\mathrm{P}}=\omega_{0}$, leading to $\omega_{\mathrm{PC}, \mathrm{NR}}=\omega_{0}$. We remind the reader that, for each photon of NR and PC generated as idler of the parametric amplification process, a seed photon is also amplified (amplified seed-AS). In the case of a deeply sub-wavelength nonlinear medium, the component of momentum in the direction of the film thickness $\left(k_{z}\right)$ does not need to be conserved, and the only relevant phase matching condition involves the in-plane wavevector components $\vec{k}_{r}$, according to

$$
\vec{k}_{r}^{(\mathrm{NR}, \mathrm{PC})}+\vec{k}_{r}^{(\mathrm{AS})}=2 \vec{k}_{r}^{(\mathrm{P})} .
$$

Since the pump beam is orthogonal to the nonlinear film, it has a null in-plane wavevector component $\left(k_{r}^{(\mathrm{P})}=0\right)$, and the above constraint simplifies to $\vec{k}_{r}^{(\mathrm{NR}, \mathrm{PC})}=-\vec{k}_{r}^{(\mathrm{AS})}$. The amplification process is coherent and the amplified seed retains the phase of the input seed. Due to the sub-wavelength nature of the nonlinear medium, the longitudinal component of the amplified seed wavevector is undetermined, and the only spatial requirement is that $\vec{k}_{r}^{(\mathrm{AS})}=\vec{k}_{r}^{(\mathrm{S})}$, which has a forward and a backward solution, as shown in Figure 1a,b. In summary, for a seed photon injected into the nonlinear medium, one photon will emerge either as the phase conjugation (PC) or negative refraction (NR) of the input seed. At the same time, another photon (amplified seed) will be generated, either in the backward $\left(\mathrm{AS}_{\mathrm{b}}\right)$ or in the forward $\left(\mathrm{AS}_{\mathrm{f}}\right)$ direction. These last two signals are overlapped to the reflected (RS) and the transmitted (TS) seed, respectively. We assume that pump and seed are co-polarised. In the ideal case described here, the amplified seed can emerge either in the backward or in the forward direction, irrespective of the generation of negative refraction or phase conjugation. We shall see below how the frequency shift of the PC and NR are linked to those of the RS and TS fields.

In previous experiments, we showed that PC and NR generated by a sub-wavelength AZO film can achieve larger than unitary internal efficiency, meaning that the PC and NR have, inside the sample, an amplitude larger than the seed [24]. Here we investigate the spectral dynamics of PC and $\mathrm{NR}$, and of the amplified seed, in similar experimental conditions. We performed the degenerate four-wave mixing (FWM) experiment using a pump and probe setup schematically shown in Figure 1c. The output of a mode-locked Ti:sapphire laser with a pulse duration of $\simeq 105 \mathrm{fs}$ pumped an optical parametric amplifier (OPA), which generated a pulse train with a wavelength tuneable in the near-IR spectral region. The OPA output was split into two arms. The pump, with high intensity, was injected at normal incidence onto the ENZ film, and was focused to a full width at half maximum beam size (FWHM) of $\simeq 460 \mu \mathrm{m}$. The seed, with a lower intensity and smaller beam spot size ( $\simeq 150 \mu \mathrm{m}$ FWHM), was overlapped with the pump coming at a small $\left(\simeq 6^{\circ}\right)$ angle to the sample normal. Both the pump and the seed were vertically polarised. The $500 \mathrm{~nm}$ AZO film was deposited on a glass substrate by 
laser pulse deposition and exhibits a real part of the relative dielectric permittivity $\Re\left[\varepsilon_{\mathrm{r}}\right]$ crossing zero at $\lambda_{\mathrm{ENZ}} \simeq 1350 \mathrm{~nm}$. We choose to excite the AZO film above the crossing point $\left(\lambda_{\mathrm{p}}=\lambda_{\mathrm{s}}=1400 \mathrm{~nm}\right)$. This choice was motivated by recent findings showing that the frequency degenerate FWM is more efficient at wavelengths longer than the $\lambda_{E N Z}[18,24]$. For the wavelength of choice, the real and imaginary part of the refractive index of the AZO sample measured by ellipsometry were $n_{\mathrm{r}} \simeq 0.3$ and $n_{\mathrm{i}} \simeq 0.89$, respectively. The pump intensity was set to $\simeq 770 \mathrm{GW} / \mathrm{cm}^{2}$. The input seed peak power was kept below $1 \mathrm{GW} / \mathrm{cm}^{2}$ through all the experiments. We recorded the power spectra of PC (Figure 2a), NR (b), reflection (c), and transmission (d) as a function of the delay between the pump and the seed ( $\tau$, where $\tau<0$ for the seed impinging on the sample before the pump pulse). From linear measurements, the reflection and transmission coefficients at the seed wavelength are $R \simeq 0.51$ and $T \simeq 0.04$. The spectra of the nonlinear signals were collected using the same spectrometer. The normalised spectrograms show that, at the delay corresponding to maximum generation efficiency, both the PC and NR are red-shifted with respect to the input seed, yet with different spectral shifts. Each spectrogram in the four panels is normalised to its maximum. The transmission and reflection spectrograms have a more complex structure that we interpret as the overlap of the signal reflection and transmission with the amplified seed field $\left(\mathrm{AS}_{\mathrm{b}}\right.$ and $\mathrm{AS}_{\mathrm{f}}$, respectively).

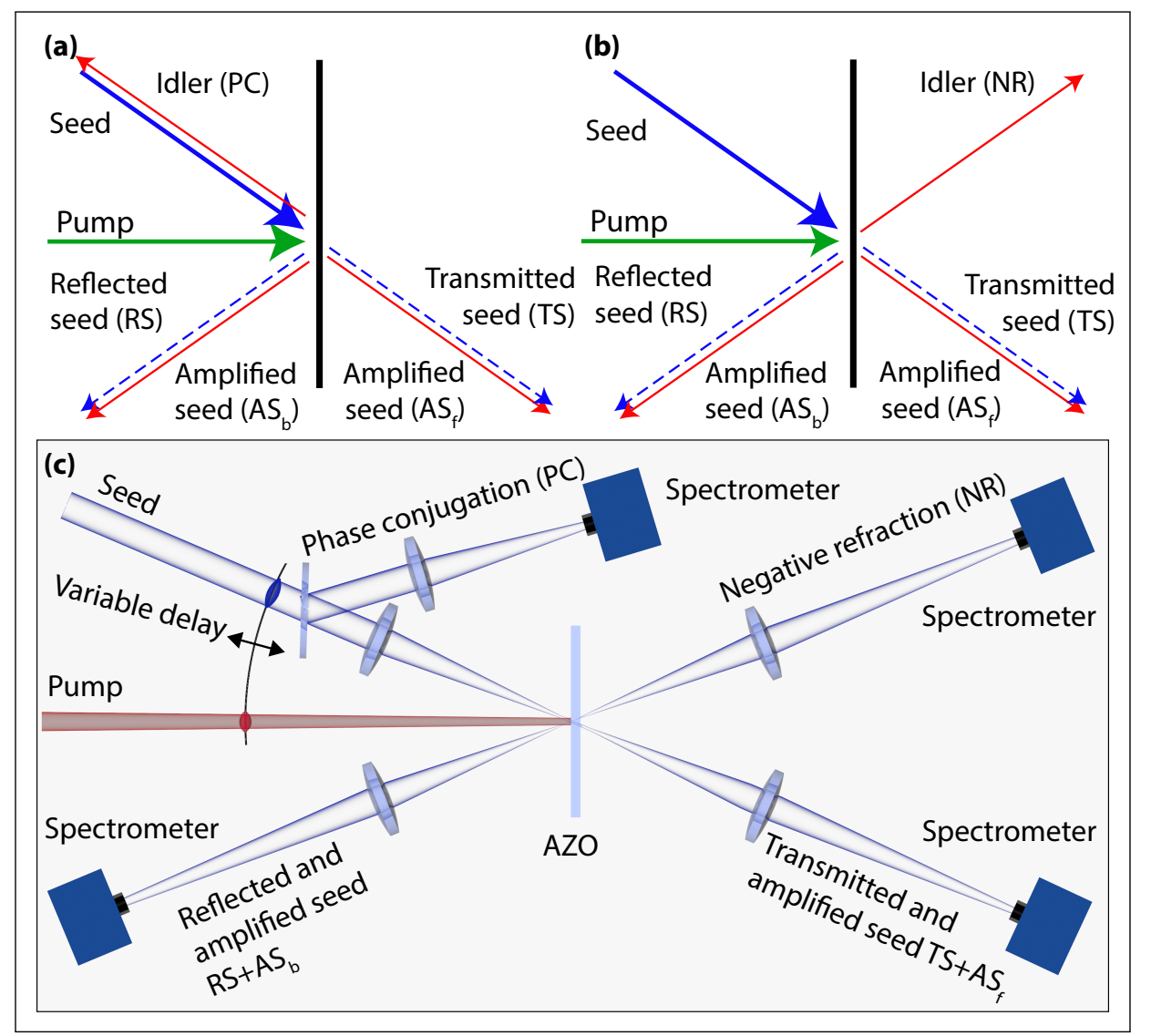

Figure 1. (a,b) describe the two four-wave mixing processes that generate the PC and NR signals, respectively. The pump beam is illustrated with a green arrow while the input seed is shown with a blue arrow. Red arrows show the result of the parametric amplification process, while blue dashed arrows show the linear reflection and transmission. For each of these two four-wave mixing processes, the seed field is amplified and emerges along both the transmitted and reflected directions. (c) Sketch of the experimental setup. Pump and seed fields have the same wavelength $(1400 \mathrm{~nm})$ and vertical polarisation. The seed is overlapped to the pump with an angle of $\simeq 6^{\circ}$. The delay between the pump and seed pulses is controlled by a delay line (not shown). A spectrometer is used to collect the radiation emerging along the direction of the seed reflection and transmission ( $\mathrm{RS}+\mathrm{AS}_{\mathrm{b}}$ and $\mathrm{TS}+\mathrm{AS}_{\mathrm{f}}$ ), as well as to characterise the PC and NR. 


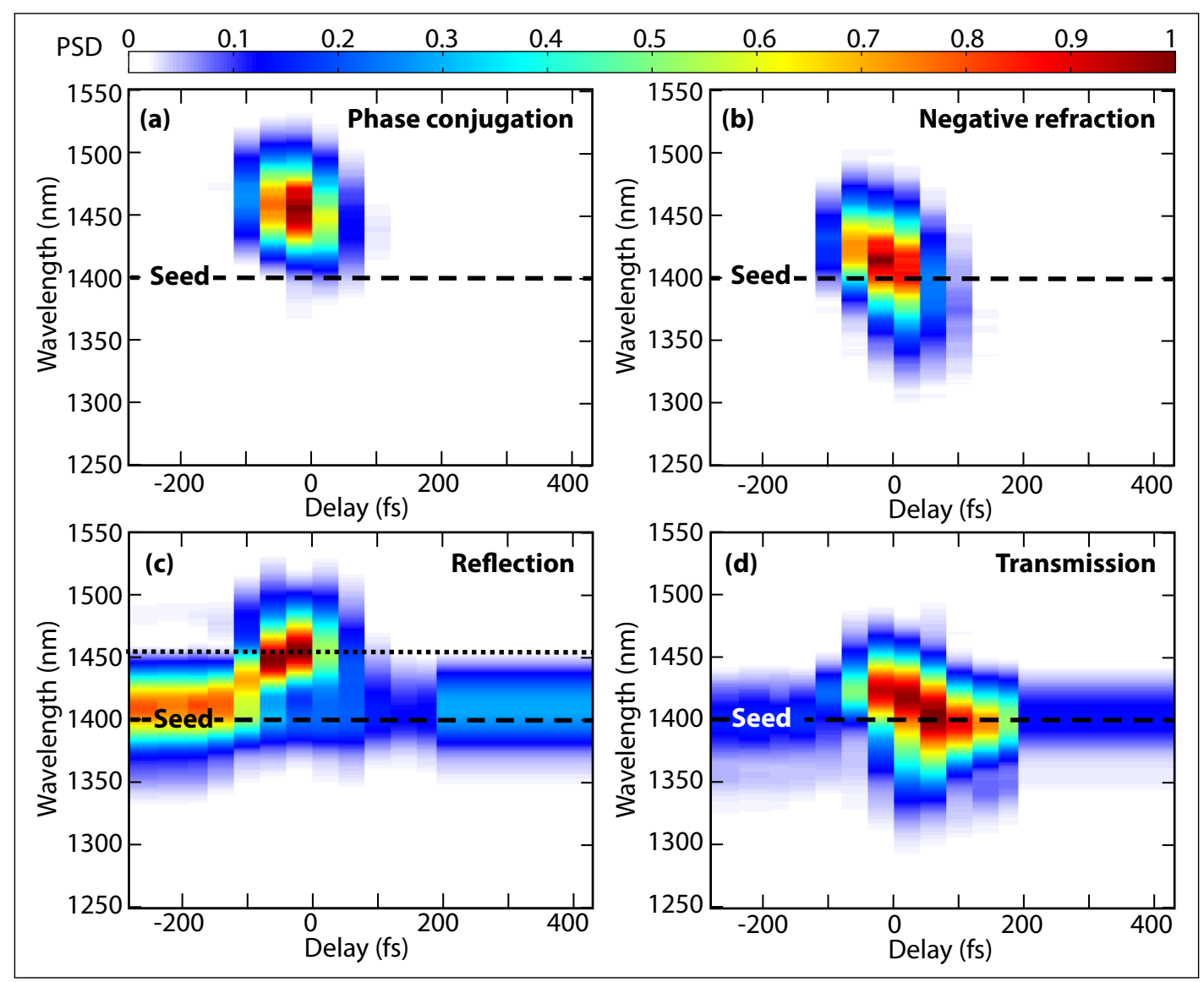

Figure 2. Measured spectrograms (normalised power spectral density (PSD)) for (a) the PC, (b) the NR, (c) the reflection, and (d) the transmission of the input seed field. See the colour bar for the PSD above panels $(\mathbf{a}, \mathbf{b})$. The horizontal axis in each panel denotes the delay between the pump and the seed pulses. The vertical axis indicates the wavelength. The dashed line is at the seed carrier wavelength $(1400 \mathrm{~nm})$, while the dotted line refers to the amplified seed measurement (see text for details).

To better understand the spectral evolution, we plot the carrier wavelength shift (blue line) as a function of the pump-probe delay for both the PC and NR signals in Figure 3a,b, respectively. We overlap the wavelength shift with the PC and NR external generation efficiencies (red). The generation efficiency is calculated as the measured signal (PC or NR) energy at a specific pump-probe delay divided by the input seed pulse energy. One can see that the PC is shifted by more than $60 \mathrm{~nm}$ with respect to the input seed wavelength, with a maximum shift occurring for the seed overlapped with the leading edge of the pump pulse. Conversely, the NR wavelength shift is larger when the seed is overlapped with either the leading or the trailing edge of the pump pulse, yet with the opposite sign. The reflected seed is expected to drop and slowly regain the original unperturbed wavelength as a function of the pump-probe delay $\tau$, as shown for an intraband excitation in [15]. This explains, for instance, why the reflection at $-200 \mathrm{fs}$ is stronger than that at $400 \mathrm{fs}$. Unlike [15], after an initial drop, the reflected signal suddenly increases at a wavelength $\simeq 60 \mathrm{~nm}$ red-shifted with respect to the input seed, as shown in Figure 3c. We interpret this as the combination of the reflection dynamics already reported in [15] with the signal generated by the parametric process in conjunction with PC and NR. We note that this shift is larger than the probe spectral bandwidth of the input seed $(\simeq 40 \mathrm{~nm})$. A similar situation can be qualitatively observed along the transmission direction. However, since the frequency shift for NR switches from positive to negative, it is impossible to completely separate the two contributions in Figure 2d. 

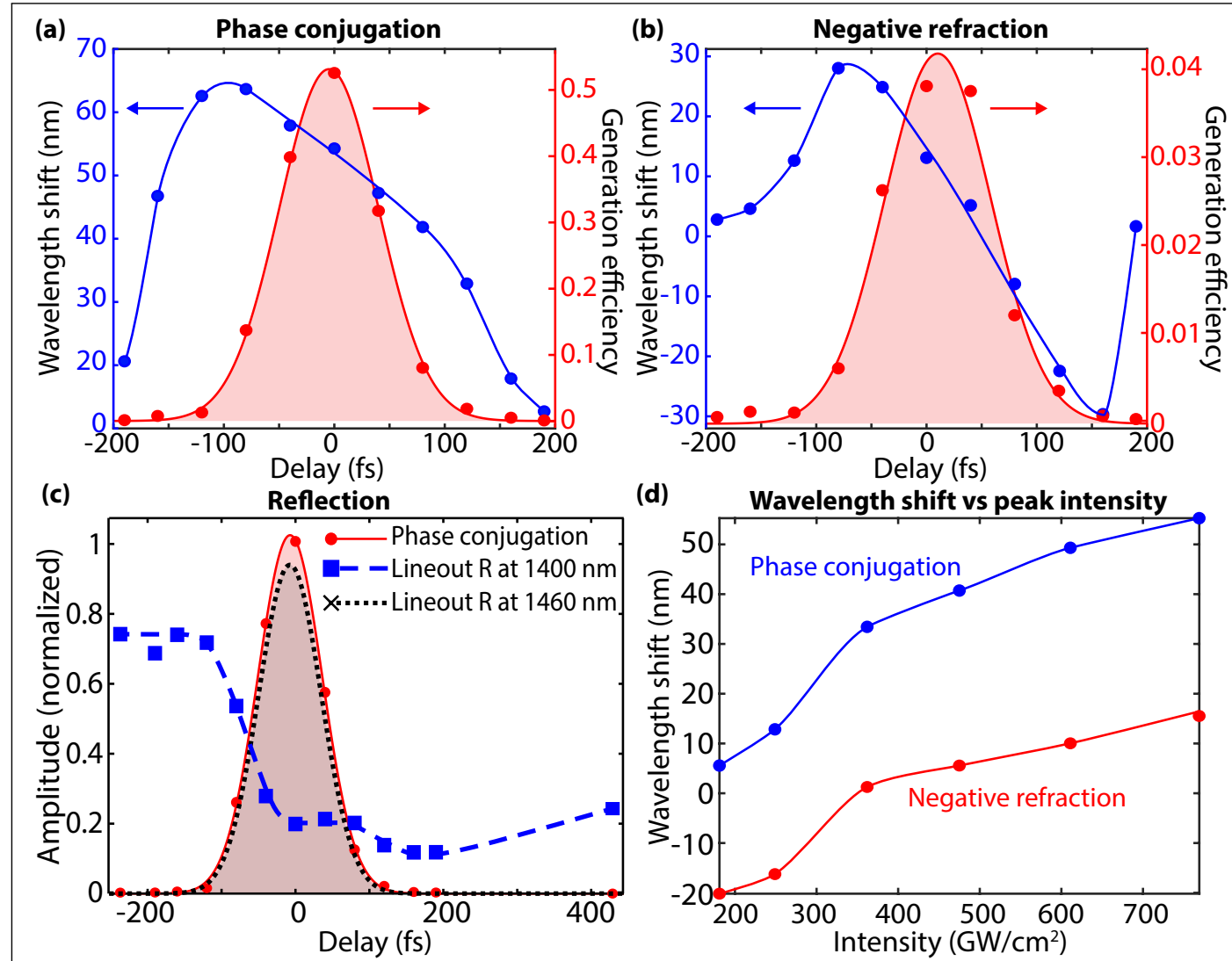

Figure 3. (a) Carrier wavelength shift of the PC signal as a function of the pump-probe delay $\Delta \tau$ (blue-curve) overlapped with the PC generation efficiency (red curve). (b) Carrier wavelength shift of the NR signal as a function of the pump-probe delay $\tau$ (blue curve) overlapped with the NR generation efficiency (red curve). (c) Reflection spectrogram as in Figure 2c, with a dashed blue line at the input seed carrier wavelength and a black dotted line at the carrier wavelength of the PC peak. (d) Measured wavelength shifts for the PC (blue) and the NR (red) nonlinear products, as a function of the pump intensity.

\section{Discussion}

The broad frequency shift observed for four-wave mixing around the epsilon-near-zero region only partially arises from the same physical mechanism responsible for the frequency shift of reflected and transmitted beams from a time-varying surface [25]. The fields generated by the nonlinear polarisation experience an adiabatic shift of the refractive index. However, a simple application of that model to our experiment would predict shifts comparable to those reported in [25], which are nearly three times smaller than what is reported here. Another contribution to the frequency shift of all beams originates from the time-varying Fresnel coefficients at the two interfaces air/AZO and $\mathrm{AZO} /$ glass. A complex refractive index that varies in time at a faster-than-picosecond time scale, as in our case, leads to significant changes in the wavelength of the impinging fields. We have estimated the magnitude of the effect that such a change would provide assuming a large change in refractive index $(\Delta n=2)$, delivered in a short, $50 \mathrm{fs}$ time period. Furthermore, even for this extreme case, the time dependence of the complex Fresnel coefficients would only account for $12 \mathrm{~nm}$ of shift in NR and $35 \mathrm{~nm}$ in PC. Furthermore, despite combining the two effects, we could only account for about $35 \%$ of the observed shift for the forward PC. We therefore speculate that the experimental results indicate possibly non-trivial underlying physical effects that might require a full microscopic model of this experimental study. 
Finally, we note that the simple model of an infinitely thin time-varying medium presented in Figure 1a,b would imply a symmetric effect in the forward and backward directions. In contrast, we observe that PC and NR have different spectral dynamics, and each of them matches that of the amplified seed along the reflection (PC) and transmission (NR). Overall, the forward signals generated by the four-wave mixing process show a spectral blue shift with respect to those generated in the backward direction, as seen in Figure 3d. This figure shows the dependence of the wavelength shift for PC (blue) and NR (red) for increasing pump intensities. The two curves follow a qualitatively similar trend, yet with a rigid blue shift of the NR that is $30 \mathrm{~nm}$ larger. The asymmetry between forward and backward directions may be attributed to the difference between the Fresnel coefficients experienced by the forward and backward propagating beams. Absorption, which induces an exponential decay of all beams (the attenuation coefficient is $\alpha=4 \pi n_{i} / \lambda=0.0079 \mathrm{~nm}^{-1}$ ), may also contribute to the observed asymmetries, as well as the cross phase modulation between the pump and the generated radiation, which mainly affects the forward propagating beam. Once again, the simple models outlined above can explain some of the difference between backward and forward, but intriguingly cannot fully account for the observed frequency shift.

\section{Conclusions}

We have experimentally investigated the frequency shift for PC and NR, and for the amplified signals generated by a four-wave mixing process in a thin film of AZO in the ENZ wavelength region. We observed extremely large wavelength shifts, exceeding $60 \mathrm{~nm}$ in the backward propagating radiation. We also recorded a different wavelength shift for the forward and backward scattered waves. Attempts to explain the observations with recently proposed models are not fully satisfactory, implying that the dynamics of nonlinear time-varying media still requires further theoretical investigation. Our results raise new questions about the fundamental physics of time-varying media in the ENZ regime and pave the way for a new range of applications in integrated nonlinear optics, e.g., for frequency conversion and optical switch. All the data supporting this manuscript are available at [30].

Author Contributions: V.B., S.V., and E.C. acquired the data, and C.D. fabricated the sample. All authors contributed to the conceptualisation of the work; V.B., S.V., D.F., and M.C. contributed to the formal analysis; M.F., A.B., V.M.S., D.F., and M.C. provided the required resources; V.B., S.V., D.F., and M.C. contributed to the original draft preparation; all authors contributed to the writing, review, and editing of the manuscript. All authors have read and agreed to the published version of the manuscript.

Funding: This research was funded by UK Research and Innovation (UKRI), Innovation Fellowship, grant number EP/S001573/1; The Engineering and Physical Sciences Research Council (EPSRC, UK), grant numbers EP/M009122/1 and EP/P006078/2; The U.S. Department of Energy, Office of Basic Energy Sciences, Division of Materials Sciences and Engineering, award number DE-SC0017717 (sample preparation); The US Air Force Office of Scientific Research (AFOSR), award number FA9550-18-1-0002; and the US Office of Naval Research (optical characterisation). The APC was funded by UKRI, through the University of Glasgow (UK).

Acknowledgments: The authors acknowledge fruitful discussions with Lucia Caspani and Jacob Khurgin.

Conflicts of Interest: The authors declare that there is no conflict of interest.

\section{References}

1. Mendonça, J.T. Theory of Photon Acceleration; CRC Press: Boca Raton, FL, USA, 2000.

2. AuYeung, J.C. Phase-conjugate reflection from a temporal dielectric boundary. Opt. Lett. 1983, 8, 148-150. [CrossRef]

3. Mendonca, J.T.; Brodin, G.; Marklund, M. Vacuum effects in a vibrating cavity: time refraction, dynamical Casimir effect, and effective Unruh acceleration. Phys. Lett. A 2008, 372, 5621-5624. [CrossRef]

4. Westerberg, N.; Cacciatori, S.; Belgiorno, F.; Dalla Piazza, F.; Faccio, D. Experimental quantum cosmology in time-dependent optical media. New J. Phys. 2014, 16, 075003. [CrossRef] 
5. Faccio, D.; Carusotto, I. Dynamical Casimir effect in optically modulated cavities. EPL (Europhys. Lett.) 2011, 96, 24006. [CrossRef]

6. Shaltout, A.; Kildishev, A.; Shalaev, V. Time-varying metasurfaces and Lorentz non-reciprocity. Opt. Mater. Express 2015, 5, 2459-2467. [CrossRef]

7. Martínez-Romero, J.S.; Becerra-Fuentes, O.; Halevi, P. Temporal photonic crystals with modulations of both permittivity and permeability. Phys. Rev. A 2016, 93, 063813. [CrossRef]

8. Prain, A.; Vezzoli, S.; Westerberg, N.; Roger, T.; Faccio, D. Spontaneous Photon Production in Time-Dependent Epsilon-Near-Zero Materials. Phys. Rev. Lett. 2017, 118, 133904. doi:10.1103/PhysRevLett.118.133904. [CrossRef]

9. Engheta, N. Pursuing near-zero response. Science 2013, 340, 286-287. [CrossRef]

10. Luk, T.S.; De Ceglia, D.; Liu, S.; Keeler, G.A.; Prasankumar, R.P.; Vincenti, M.A.; Scalora, M.; Sinclair, M.B.; Campione, S. Enhanced third harmonic generation from the epsilon-near-zero modes of ultrathin films. Appl. Phys. Lett. 2015, 106, 151103. [CrossRef]

11. Liberal, I.; Engheta, N. Near-zero refractive index photonics. Nat. Photonics 2017, 11, 149. [CrossRef]

12. Capretti, A.; Wang, Y.; Engheta, N.; Dal Negro, L. Comparative study of second-harmonic generation from epsilon-near-zero indium tin oxide and titanium nitride nanolayers excited in the near-infrared spectral range. ACS Photonics 2015, 2, 1584-1591. [CrossRef]

13. Kinsey, N.; DeVault, C.; Kim, J.; Ferrera, M.; Shalaev, V.; Boltasseva, A. Epsilon-near-zero Al-doped ZnO for ultrafast switching at telecom wavelengths. Optica 2015, 2, 616-622. [CrossRef]

14. Alam, M.Z.; De Leon, I.; Boyd, R.W. Large optical nonlinearity of indium tin oxide in its epsilon-near-zero region. Science 2016, 352, 795-797. [CrossRef] [PubMed]

15. Caspani, L.; Kaipurath, R.; Clerici, M.; Ferrera, M.; Roger, T.; Kim, J.; Kinsey, N.; Pietrzyk, M.; Di Falco, A.; Shalaev, V.M.; et al. Enhanced nonlinear refractive index in $\varepsilon$-near-zero materials. Phys. Rev. Lett. 2016, 116, 233901. [CrossRef]

16. Clerici, M.; Kinsey, N.; DeVault, C.; Kim, J.; Carnemolla, E.G.; Caspani, L.; Shaltout, A.; Faccio, D.; Shalaev, V.; Boltasseva, A.; et al. Controlling hybrid nonlinearities in transparent conducting oxides via two-colour excitation. Nat. Commun. 2017, 8, 15829. [CrossRef]

17. Yang, Y.; Kelley, K.; Sachet, E.; Campione, S.; Luk, T.S.; Maria, J.P.; Sinclair, M.B.; Brener, I. Femtosecond optical polarization switching using a cadmium oxide-based perfect absorber. Nat. Photonics 2017, 11, 390. [CrossRef]

18. Carnemolla, E.G.; Caspani, L.; DeVault, C.; Clerici, M.; Vezzoli, S.; Bruno, V.; Shalaev, V.M.; Faccio, D.; Boltasseva, A.; Ferrera, M. Degenerate optical nonlinear enhancement in epsilon-near-zero transparent conducting oxides. Opt. Mater. Express 2018, 8, 3392-3400. [CrossRef]

19. Niu, X.; Hu, X.; Chu, S.; Gong, Q. Epsilon-Near-Zero Photonics: A New Platform for Integrated Devices. Adv. Opt. Mater. 2018, 6, 1701292. [CrossRef]

20. Wood, M.G.; Campione, S.; Parameswaran, S.; Luk, T.S.; Wendt, J.R.; Serkland, D.K.; Keeler, G.A. Gigahertz speed operation of epsilon-near-zero silicon photonic modulators. Optica 2018, 5, 233-236. [CrossRef]

21. Reshef, O.; De Leon, I.; Alam, M.Z.; Boyd, R.W. Nonlinear optical effects in epsilon-near-zero media. Nat. Rev. Mater. 2019, 4, 535-551. [CrossRef]

22. Shaltout, A.M.; Shalaev, V.M.; Brongersma, M.L. Spatiotemporal light control with active metasurfaces. Science 2019, 364, doi:10.1126/science.aat3100. [CrossRef] [PubMed]

23. Kinsey, N.; DeVault, C.; Boltasseva, A.; Shalaev, V.M. Near-zero-index materials for photonics. Nat. Rev. Mater. 2019, 1-19. [CrossRef]

24. Vezzoli, S.; Bruno, V.; DeVault, C.; Roger, T.; Shalaev, V.M.; Boltasseva, A.; Ferrera, M.; Clerici, M.; Dubietis, A.; Faccio, D. Optical Time Reversal from Time-Dependent Epsilon-Near-Zero Media. Phys. Rev. Lett. 2018, 120, 043902. doi:10.1103/PhysRevLett.120.043902. [CrossRef] [PubMed]

25. Khurgin, J.B.; Clerici, M.; Bruno, V.; Caspani, L.; DeVault, C.; Kim, J.; Shaltout, A.; Boltasseva, A.; Shalaev, V.M.; Ferrera, F.; Faccio, D.; Kinsey, N. Adiabatic frequency shifting in epsilon near zero materials: The role of group velocity. arXiv 2019, arXiv:1906.04849.

26. Pendry, J. Time reversal and negative refraction. Science 2008, 322, 71-73. [CrossRef]

27. Sivan, Y.; Pendry, J.B. Theory of wave-front reversal of short pulses in dynamically tuned zero-gap periodic systems. Phys. Rev. A 2011, 84, 033822. [CrossRef] 
28. Palomba, S.; Zhang, S.; Park, Y.; Bartal, G.; Yin, X.; Zhang, X. Optical negative refraction by four-wave mixing in thin metallic nanostructures. Nat. Mater. 2012, 11, 34. [CrossRef]

29. Rao, S.M.; Lyons, A.; Roger, T.; Clerici, M.; Zheludev, N.I.; Faccio, D. Geometries for the coherent control of four-wave mixing in graphene multilayers. Sci. Rep. 2015, 5, 15399. [CrossRef]

30. Broad Frequency Shift of Parametric Processes in Epsilon-Near-Zero Time-Varying Media. Available online: http:/ / researchdata.gla.ac.uk/937/ (accessed on 13 February 2020).

(C) 2020 by the authors. Licensee MDPI, Basel, Switzerland. This article is an open access article distributed under the terms and conditions of the Creative Commons Attribution (CC BY) license (http://creativecommons.org/licenses/by/4.0/). 